\title{
New Eight Dimensional Hyperchaotic Cryptosystem
}

S. N. Lagmiri ${ }^{\# 1}$, N. Elalami ${ }^{\# 2}$, J. Elalami ${ }^{\# 3}$
\#1 Information and Production System,Mohammadia School Engineering, Mohamed V
University in Rabat, Morocco
\#2 LAII,Mohammadia School Engineering,Mohamed V University in Rabat, Morocco
\#3 LASTIMI,Higher School of Technology of Sale, Mohamed V University in Rabat,
Morocco

\section{ABSTRACT}

The use of hyperchaotic signals to obtain highly secure communication is increasingly studied. This class of system has more parameters and more complex attractors than regular chaotic system which makes the system is strong in secure data transmission. In this paper, a new eight dimensional hyperchaotic system is introduced and analyzed. Basic dynamical analysis of this new system is carried out by means of system equilibria, sensitivity to initial conditions and dissipativity. In addition, in this paper Lyapunovexponents spectrum of the proposed system has been executed by means of specific parameters and initial conditions. In order to overcome the problems of synchronization of this eight hyperchaotic system, a high gain observer is used and this method of control give many advantages such as easy implementation, high accuracy and stable performance.

The results of Matlab simulation using the chaotic masking scheme, show that the restoredsignal of the realized system is identical to generated one. The purpose of this research work is to use this hyperchaotic generator in secure communication field.

Keywords:Hyperchaos, High gain observer, Synchronization, Chaotic masking.

\section{Corresponding Author:S.N.LAGMIRI(SouadNajoua LAGMIRI)}

\section{INTRODUCTION}

Chaotic systems are nonlinear dynamical systems which are an aperiodic, deterministic and sensitive to small changes to initial conditions and control parameters [1]. The Lyapunov exponent is a measure of the divergence of phase points that are initially very close and can be used to quantify the behavior of chaotic systems. A positive Lyapunov exponent and phase space compactness are usually taken as defining conditions for a chaotic system. 
In the last four decades, there is a great deal of interest in the chaos literature in modeling and analysis of new chaotic systems. Many chaotic systems were introduced such as Rössler system, Qi system, Chen system and others. With the rapid development of chaotic theory, the design of hyperchaotic system and its circuit realization problems are considered [3, 4].Hyperchaotic system is a chaotic system with more than one positive Lyapunov exponent,meaning that the chaotic dynamics of the system are expanded in more than one direction leading to more complex attractors [7].Those systems have several important applications in science and engineering such as oscillators [18, 20], cryptosystems [8, 12], secure communications $[5,6,9]$.

The problem of control of a chaotic system is to find a state feedback control law to stabilize a chaotic system around its unstable equilibrium [10].Some popular methods for chaos control are active control [21], adaptive control [17], sliding mode control [15], etc.

Major works on synchronization of chaotic systems deal with the synchronization by high gain observer [11] which has the goal of using the output of the mastersystem to control the slave system so that the output of the slave system tracks theoutput of the master system asymptotically[2].

In this paper, we present the approach to secure communications by using a new eight dimensional hyperchaotic system and based on the theory of high gain observers.

This paper is organized as follows: section 2 introduces the model of proposed eightdimensionalhyperchaoticsystem. Theanalyze of this system is presented in section 3, implementation and experimental results of synchronization and chaotic masking are appeared respectively in section 4 and 5, and section 6 discussesconclusion.

\section{EIGHT DIMENSIONAL HYPERCHAOTIC SYSTEM}

In this section, we present the mathematical model of the eight dimensional hyperchaotic system defined by:

$$
\left\{\begin{array}{l}
\dot{x}_{1}=-10 x_{1}+15 x_{5}-x_{3} x_{5} x_{6} x_{8} \\
\dot{x}_{2}=0.5 x_{2}-25 x_{6}+x_{1} x_{4} x_{5} x_{8} \\
\dot{x}_{3}=-15 x_{3}+15 x_{8}-0.1 x_{1} x_{2} x_{4} x_{6} \\
\dot{x}_{4}=-10 x_{4}+x_{1} x_{2} x_{3} x_{5} \\
\dot{x}_{5}=20 x_{5}+10 x_{7}-x_{1} x_{2} x_{3} x_{6} \\
\dot{x}_{6}=-10 x_{6}+10 x_{5}+x_{2} x_{3} x_{4} x_{7} \\
\dot{x}_{7}=5 x_{7}+4 x_{2}-1.5 x_{1} x_{4} x_{5} x_{8} \\
\dot{x}_{8}=-15 x_{8}+x_{1}-x_{3} x_{4} x_{5} x_{6}
\end{array}\right.
$$

Where $x=\left(x_{1}, x_{2}, \ldots, x_{n}\right)^{T} \in R^{n}$ is the state vector.

\section{ANALYSIS OF THE NOVEL HYPERCHAOTIC SYSTEM}

This section gives the qualitative properties of the eight dimensional hyperchaotic system proposed in this research work.

\subsection{Dissipativity}

We write the system (1) in vector notation as [16]: 


$$
\dot{x}=f(x)=\left[\begin{array}{l}
f_{1}(x) \\
f_{2}(x) \\
f_{3}(x) \\
f_{4}(x) \\
f_{5}(x) \\
f_{6}(x) \\
f_{7}(x) \\
f_{8}(x)
\end{array}\right]
$$

The divergence of the vector field $f$ on $R^{n}$ is obtained as:

$$
\begin{aligned}
\operatorname{div} f=\frac{\partial f_{1}(x)}{\partial x_{1}}+\frac{\partial f_{2}(x)}{\partial x_{2}}+\frac{\partial f_{3}(x)}{\partial x_{3}} & +\frac{\partial f_{4}(x)}{\partial x_{4}}+\frac{\partial f_{5}(x)}{\partial x_{5}}+\frac{\partial f_{6}(x)}{\partial x_{6}}+\frac{\partial f_{7}(x)}{\partial x_{7}}+\frac{\partial f_{8}(x)}{\partial x_{8}} \\
=-10+0.5-15- & 10+20-10+5-15 \\
= & -34,5<0
\end{aligned}
$$

when $\operatorname{div} f<0$, the system (1) is dissipative.

\subsection{Equilibria and Lyapunov Exponents}

The equilibrium points of the novel hyperchaotic system (1) are obtained by solving the nonlinear equations [14]:

$$
\left\{\begin{array}{r}
-10 x_{1}+15 x_{5}-x_{3} x_{5} x_{6} x_{8}=0 \\
0.5 x_{2}-25 x_{6}+x_{1} x_{4} x_{5} x_{8}=0 \\
-15 x_{3}+15 x_{8}-0.1 x_{1} x_{2} x_{4} x_{6}=0 \\
-10 x_{4}+x_{1} x_{2} x_{3} x_{5}=0 \\
20 x_{5}+10 x_{7}-x_{1} x_{2} x_{3} x_{6}=0 \\
-10 x_{6}+10 x_{5}+x_{2} x_{3} x_{4} x_{7}=0 \\
5 x_{7}+4 x_{2}-1.5 x_{1} x_{4} x_{5} x_{8}=0 \\
-15 x_{8}+x_{1}-x_{3} x_{4} x_{5} x_{6}=0
\end{array}\right.
$$

The new hyperchaotic system (1) has unique equilibrium point $E_{0}(0,0,0,0,0,0,0,0)$.

For this latter, it's is linearized and the Jacobian matrix is given by:

$$
J=\left[\begin{array}{cccccccc}
-10 & 0 & 0 & 0 & 0 & 0 & 0 & 0 \\
0 & 0.5 & 0 & 0 & 0 & 0 & 0 & 0 \\
0 & 0 & -15 & 0 & 0 & 0 & 0 & 0 \\
0 & 0 & 0 & -10 & 0 & 0 & 0 & 0 \\
0 & 0 & 0 & 0 & 20 & 0 & 0 & 0 \\
0 & 0 & 0 & 0 & 0 & -10 & 0 & 0 \\
0 & 0 & 0 & 0 & 0 & 0 & 5 & 0 \\
0 & 0 & 0 & 0 & 0 & 0 & 0 & -15
\end{array}\right]
$$

To gain its eigenvalues, let $\left|\lambda_{i}-J_{0}\right|=0$, then the eigenvalues corresponding to equilibrium will be obtained as follows: 


$$
\begin{gathered}
\lambda_{1}=-9.999627 \lambda_{2}=4.999412 \lambda_{3}=-14.995362, \\
\lambda_{4}=-9.999725 \lambda_{5}=20.000900 \lambda_{6}=0.497939, \\
\lambda_{7}=0.616145 \lambda_{8}=1.686612
\end{gathered}
$$

Where $\lambda_{2}, \lambda_{5}, \lambda_{6}, \lambda_{7}, \lambda_{8}$ are positive real roots and $\lambda_{1}, \lambda_{3}, \lambda_{4}$ are negative real roots.

Therefore, equilibrium $\mathrm{E}_{0}$ is unstable.

The system (1) has five positive lyapunov exponents, which shows that the system is not only chaotic but also hyperchaotic.

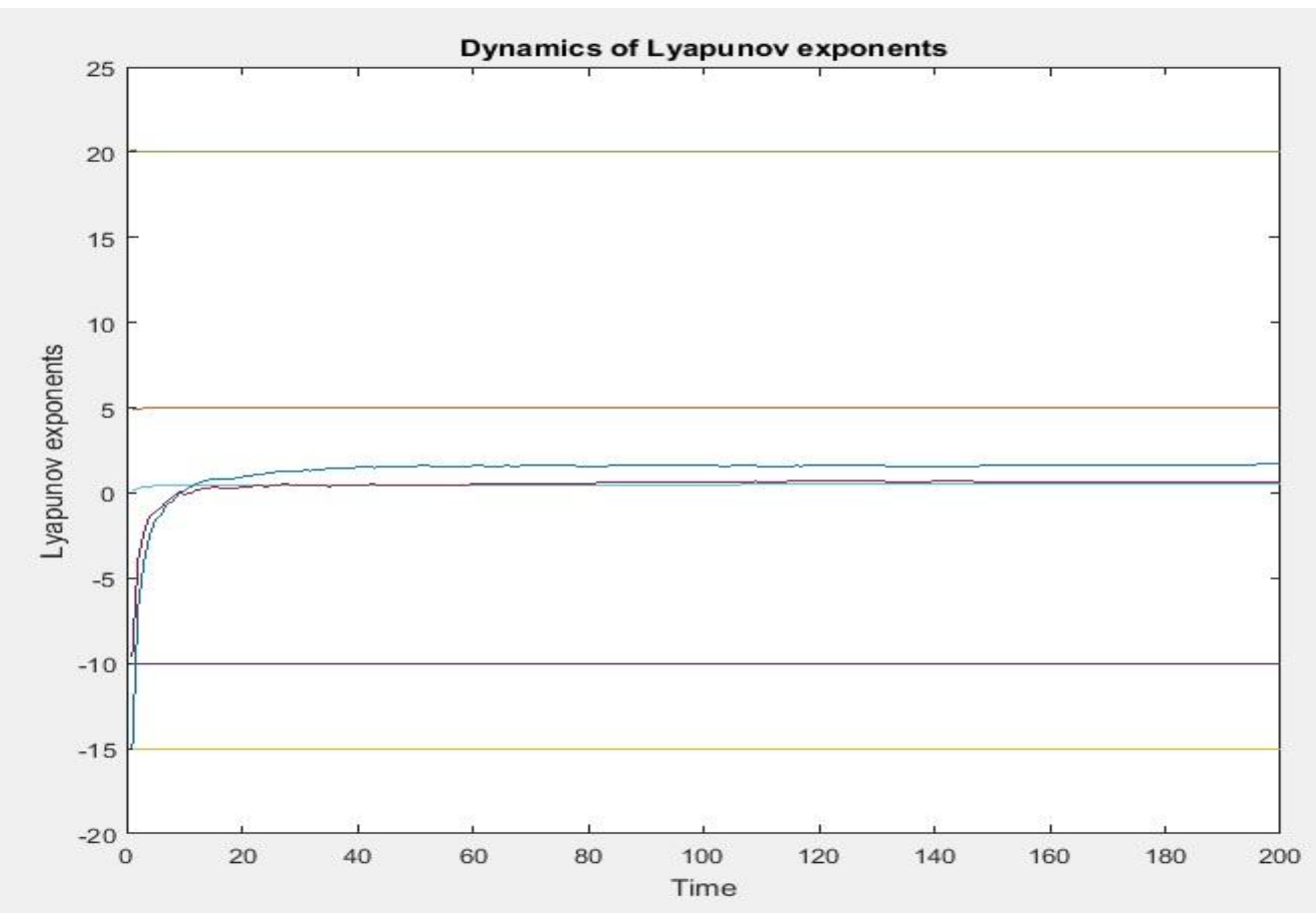

Fig 1: Dynamics of the Lyapunov exponents of the eight dimensional hyperchaoticsystem

\section{SYNCHRONIZATION OF THE NEW EIGHT HYPRCHAOTIC SYSTEM}

\subsection{Control via a high gain observer}

The problem of observer design naturally arises in a system approach, as soon as one needs unmeasured internal information from external measurements. As we know, it is almost impossible to measure all the elements of the state vector in practice (e.g., the unknown state variables, fault signals, etc).

State observers are able to provide a continuous estimation of some signals which are not measured by hardware sensors. They need a mathematical model of the process and hardware measurements of some other signals. An observer is a dynamic system whose input includes 
the control $u$ and the output $y$ and whose output is an estimate of the state vector $\hat{x}$ as it's shown in fig. 2 [19].

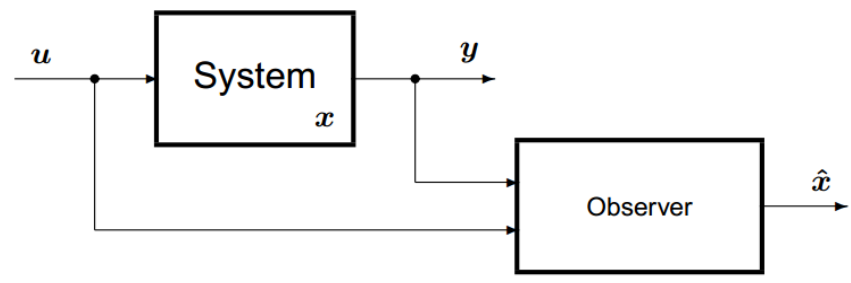

Fig 2: Principle of the observer

We consider a general representation of the hyperchaotic system as follow:

$$
\left\{\begin{array}{c}
\dot{x}=f(x) \\
y=C x
\end{array}\right.
$$

Where:

- $\quad C=\left(\begin{array}{llll}1 & 0 & \ldots & 0\end{array}\right)$ and $x \in R^{n}$ is the vector of states, $x=\left(x_{1}, x_{2}, \ldots, x_{n}\right)^{T}$.

- $f: R^{n} \rightarrow R^{n}$ is the nonlinear part of the system.

- $y \in R^{m}$ is the system measured output with $m<n$, and

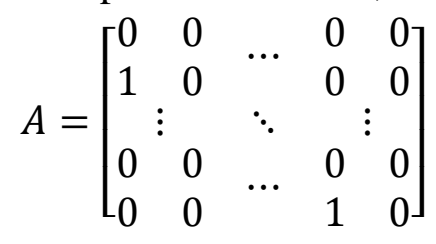

It is necessary the design of an auxiliary system so called observer system to reconstruct then known states or unmeasurables. Firstly, we give necessary and sufficient conditions to establish whether the system (3) is observable.

Now, consider the following assumptions [22]:

\section{A. Assumptions}

A1.The system given in Eqs (3) is locally uniformly observable (Gauthier et al., 1992), hence for all $x \in R^{n}$, satisfies the observability rank condition:

$$
\operatorname{rang}\left\{\frac{\partial}{\partial x} \vartheta\right\}=n
$$

Here $\vartheta$ is the observability vector function define $\mathrm{d} \vartheta=\left(d L_{f}^{0} h, d L_{f}^{1} h, \ldots, d L_{f}^{n-1} h\right)^{T} L_{f}^{r}$ the rorder Lie derivatives, which are the directional derivatives of the corresponding state variables along the measured output trajectory. And $d L_{f}^{r} h$ are the differentials of the $\mathrm{r}^{\text {th }}$-order Lie derivatives defined recursively as follows:

$$
L_{f}^{0} h:=h, d L_{f}^{0} h:=d h=\left(\frac{\partial h}{\partial x_{1}}, \ldots, \frac{\partial h}{\partial x_{n}}\right)
$$




$$
\begin{gathered}
L_{f}^{1} h:=<d h, f>=\sum_{i=1}^{n} \frac{\partial h}{\partial x_{i}} f_{i} \\
d L_{f}^{1} h:=\left(\frac{\partial}{\partial x_{1}}\left(\sum_{i=1}^{n} \frac{\partial h}{\partial x_{i}} f_{i}\right), \ldots, \frac{\partial}{\partial x_{n}}\left(\sum_{i=1}^{n} \frac{\partial h}{\partial x_{i}} f_{i}\right)\right) \\
L_{f}^{r} h:=<d L_{f}^{r-1} h, f>=L_{f}\left(L_{f}^{r-1} h\right), \quad r \geq 2
\end{gathered}
$$

A2. All the trajectories $x\left(t, x_{0}\right), x_{0} \in R^{n}$ of the system (3) are bounded.

Considering the set $\Omega \mathrm{c} R^{n}$ as the corresponding physically realizable domain, such that:

$\Omega=\left\{\left(x_{i}\right)_{i=1}^{n} \in R_{+}^{n} / 0 \leq x_{i} \leq x_{\max }\right\}$

In most practical cases, $\Omega$ will be an open connected relatively compact subset of $R^{n}$, and in the ideal cases, $\Omega$ will be positively invariant under the dynamic (3).

In order to analyze the estimation error $\varepsilon=y-x$ we consider the next assumption.

A3. The nonlinear difference vector function $\Delta \emptyset=f(x)-f(\hat{x})$ is Lipschitz bounded i.e. $\Delta \emptyset<\Lambda|\varepsilon|$.

Condition A3 can be fulfilled satisfied if the following supremumis finite:

$\Lambda:=\sup _{x \in \Omega}\left\|f^{\prime}(x)\right\|$

Where $f^{\prime}(x)$ is the Jacobian and $\|$.$\| is the matrix norm associated with the Euclidian vector$ norm.

\section{B. Propositions}

For any initial condition $x(0) \in D$ and any $\hat{x}(0) \in D$ and for $\theta$ large enough the system (1) satisfying previous assumptions [10] [11], can be estimated by the following dynamical system:

$$
\dot{\hat{x}}=f(\hat{x})-\theta * d_{\theta}^{-1} * S_{1}^{-1} * C^{\prime}(C \hat{x}-y)
$$

Where $S_{1}$ is the symetric positive definite solution of the algebraic equation:

$$
\theta S_{\theta}+A^{\prime} S_{\theta}+S_{\theta} A-C^{\prime} C=0
$$

For $\theta=1$ and it can be expressed as

$$
S_{1}(i, j)=(-1)^{i+j} C_{i+j-2}^{j-1}, \text { for } 1 \leq i, j \leq n
$$

where $C_{j}^{i}=\frac{j !}{i !(j-i) !}$

$d_{\theta}$ is a diagonal matrix defined by: 


$$
d_{\theta}=\operatorname{diag}\left(1, \frac{1}{\theta}, \ldots, \frac{1}{\theta^{n}}\right)
$$

\section{Lemma}

For $\theta$ large enough the slave system below is an observer for the drive system [13]:

$$
\dot{\hat{x}}=f(\hat{x})-\theta * d_{\theta}^{-1} * S_{1}^{-1} * C^{\prime}(C \hat{x}-z)
$$

\subsection{Synchronization problem formulation}

As reported in the literature, synchronization of hyperchaotic systems suggests the possibility for communication using hyperchaotic waveforms as carriers, perhaps with application to secure communication. The obvious approach uses ahyperchaotic system as the transmitter and a synchronous hyperchaotic system for the receiver, and several designs have been suggested that fit within this construct [8]. The variation in these designs lies in the methods for injecting an information signal at the transmitter and recovering it at the receiver.

Definition: Two hyperchaotic systems are completely synchronized if the error:

$$
\|\hat{x}-x\| \rightarrow 0 \text { as } t \rightarrow \infty
$$

It means that each state in the slave system is identical or is very close to its corresponding state in the master system along the time.

Chaos synchronization as stabilization consists in finding a control command that leads the trajectories of a dynamical error system to the origin [14].

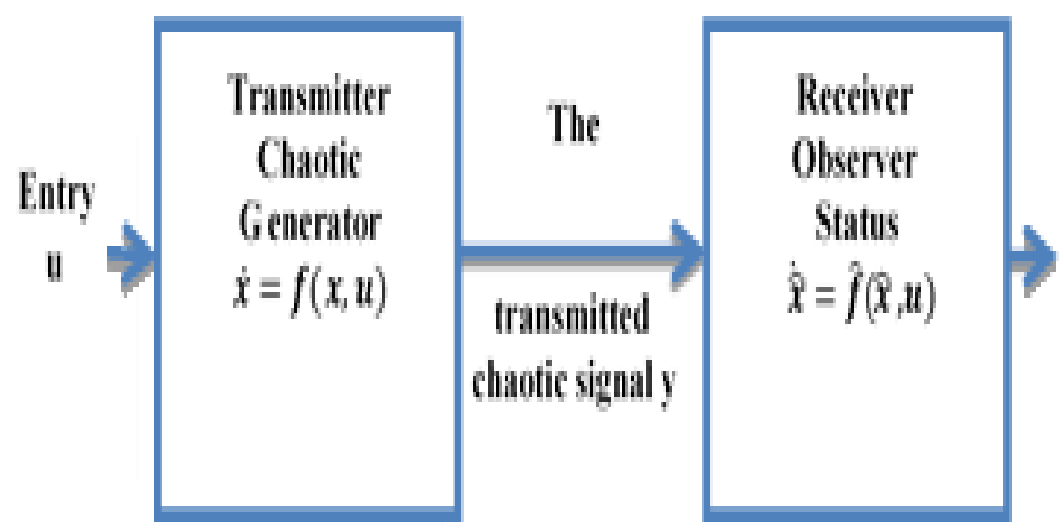

Fig 3: Principle of Master Slave synchronization

Now, let's design a high observer design for the synchronization of our 8D hyperchaotic system.

The dynamic of the high gain observer of the system (1) is given by: 


$$
\left\{\begin{array}{l}
\dot{\hat{x}}_{1}=-10 \hat{x}_{1}+15 \hat{x}_{5}-\hat{x}_{3} \hat{x}_{5} \hat{x}_{6} \hat{x}_{8}-\theta * d_{\theta}^{-1} * S_{1}^{-1} * C^{\prime} C\left(\hat{x}_{1}-x_{1}\right) \\
\dot{\hat{x}}_{2}=0.5 \hat{x}_{2}-25 \hat{x}_{6}+\hat{x}_{1} \hat{x}_{4} \hat{x}_{5} \hat{x}_{8}-\theta * d_{\theta}^{-1} * S_{1}^{-1} * C^{\prime} C\left(\hat{x}_{2}-x_{2}\right) \\
\dot{\hat{x}}_{3}=-15 \hat{x}_{3}+15 \hat{x}_{8}-0.1 \hat{x}_{1} \hat{x}_{2} \hat{x}_{4} \hat{x}_{6}-\theta * d_{\theta}^{-1} * S_{1}^{-1} * C^{\prime} C\left(\hat{x}_{3}-x_{3}\right) \\
\dot{\hat{x}}_{4}=-10 \hat{x}_{4}+\hat{x}_{1} \hat{x}_{2} \hat{x}_{3} \hat{x}_{5}-\theta * d_{\theta}^{-1} * S_{1}^{-1} * C^{\prime} C\left(\hat{x}_{4}-x_{4}\right) \\
\dot{\hat{x}}_{5}=20 \hat{x}_{5}+10 \hat{x}_{7}-\hat{x}_{1} \hat{x}_{2} \hat{x}_{3} \hat{x}_{6}-\theta * d_{\theta}^{-1} * S_{1}^{-1} * C^{\prime} C\left(\hat{x}_{5}-x_{5}\right) \\
\dot{\hat{x}}_{6}=-10 \hat{x}_{6}+10 \hat{x}_{5}+\hat{x}_{2} \hat{x}_{3} \hat{x}_{4} \hat{x}_{7}-\theta * d_{\theta}^{-1} * S_{1}^{-1} * C^{\prime} C\left(\hat{x}_{6}-x_{6}\right) \\
\dot{\hat{x}}_{7}=5 \hat{x}_{7}+4 \hat{x}_{2}-1.5 \hat{x}_{1} \hat{x}_{4} \hat{x}_{5} \hat{x}_{8}-\theta * d_{\theta}^{-1} * S_{1}^{-1} * C^{\prime} C\left(\hat{x}_{7}-x_{\grave{e}}\right) \\
\dot{\hat{x}}_{8}=-15 \hat{x}_{8}+\hat{x}_{1}-\hat{x}_{3} \hat{x}_{4} \hat{x}_{5} \hat{x}_{6}-\theta * d_{\theta}^{-1} * S_{1}^{-1} * C^{\prime} C\left(\hat{x}_{8}-x_{8}\right)
\end{array}\right.
$$

For the synchronization, the error $e$ is defined as:

$$
\begin{gathered}
e_{1}=\hat{x}_{1}-x_{1}, e_{2}=\hat{x}_{2}-x_{2}, e_{3}=\hat{x}_{3}-x_{3}, e_{4}=\hat{x}_{4}-x_{4}, e_{5}=\hat{x}_{5}-x_{5}, e_{6}=\hat{x}_{6}-x_{6}, e_{7}= \\
\hat{x}_{7}-x_{7}, e_{8}=\hat{x}_{8}-x_{8}
\end{gathered}
$$

\subsection{Synchronization results}

For simulations, the initial conditions of the 8Dhyperchaotic system(1) are chosen as:

$$
x_{1}=0 \quad x_{2}=1 \quad x_{3}=0 \quad x_{4}=2 \quad x_{5}=0 x_{6}=4 \quad x_{7}=0 \quad x_{8}=-2
$$

Also, the initial conditions of the observer(7) arechosen as:

$$
\hat{x}_{1}=2 \hat{x}_{2}=-1 \quad \hat{x}_{3}=4 \quad \hat{x}_{4}=-3 \quad \hat{x}_{5}=2 \hat{x}_{6}=-3 \hat{x}_{7}=1 \hat{x}_{8}=0
$$
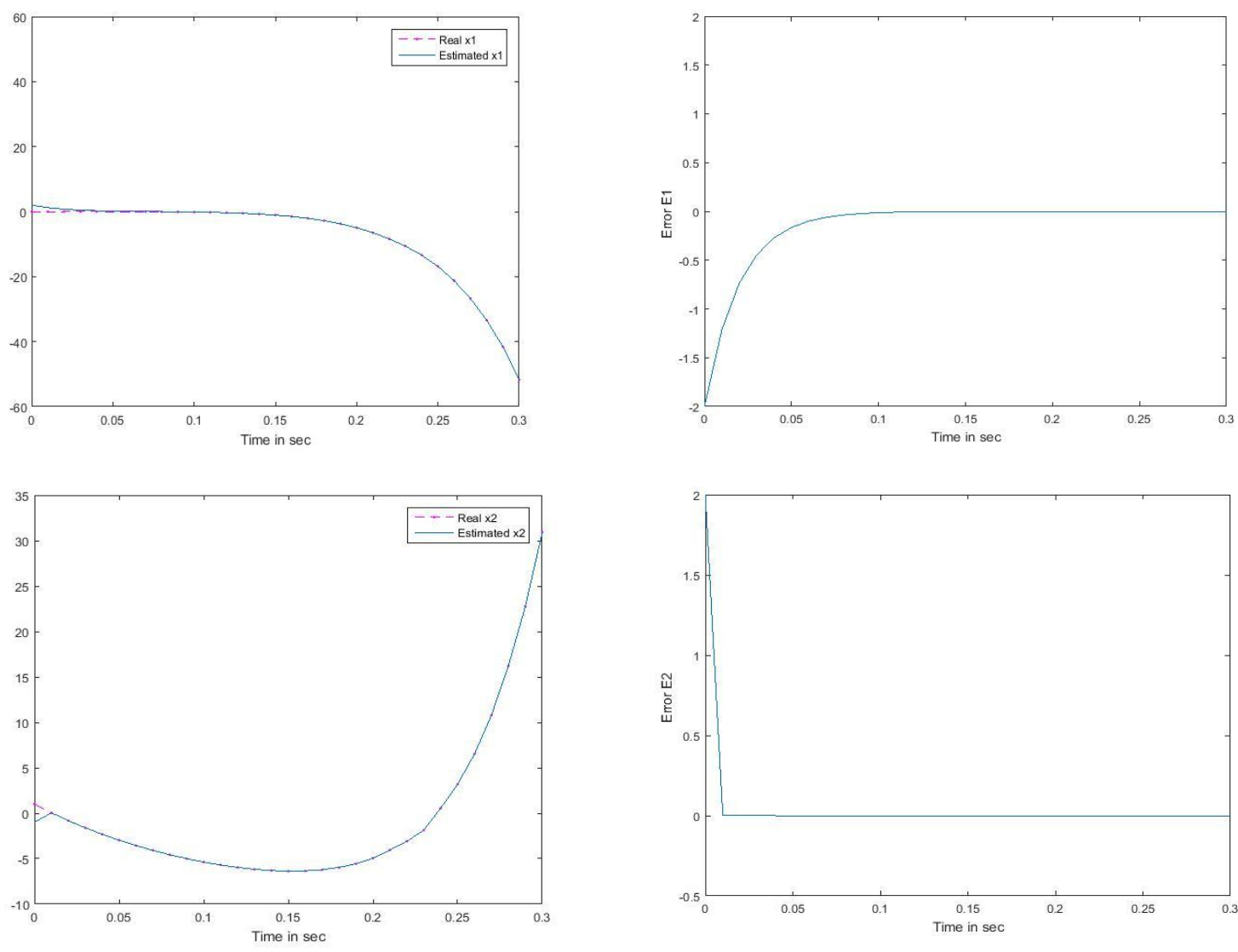
DOI: https://dx.doi.org/10.26808/rs.ca.i7v5.03 International Journal of Computer Application (2250-1797)

Volume 7- No.5, September-October 2017
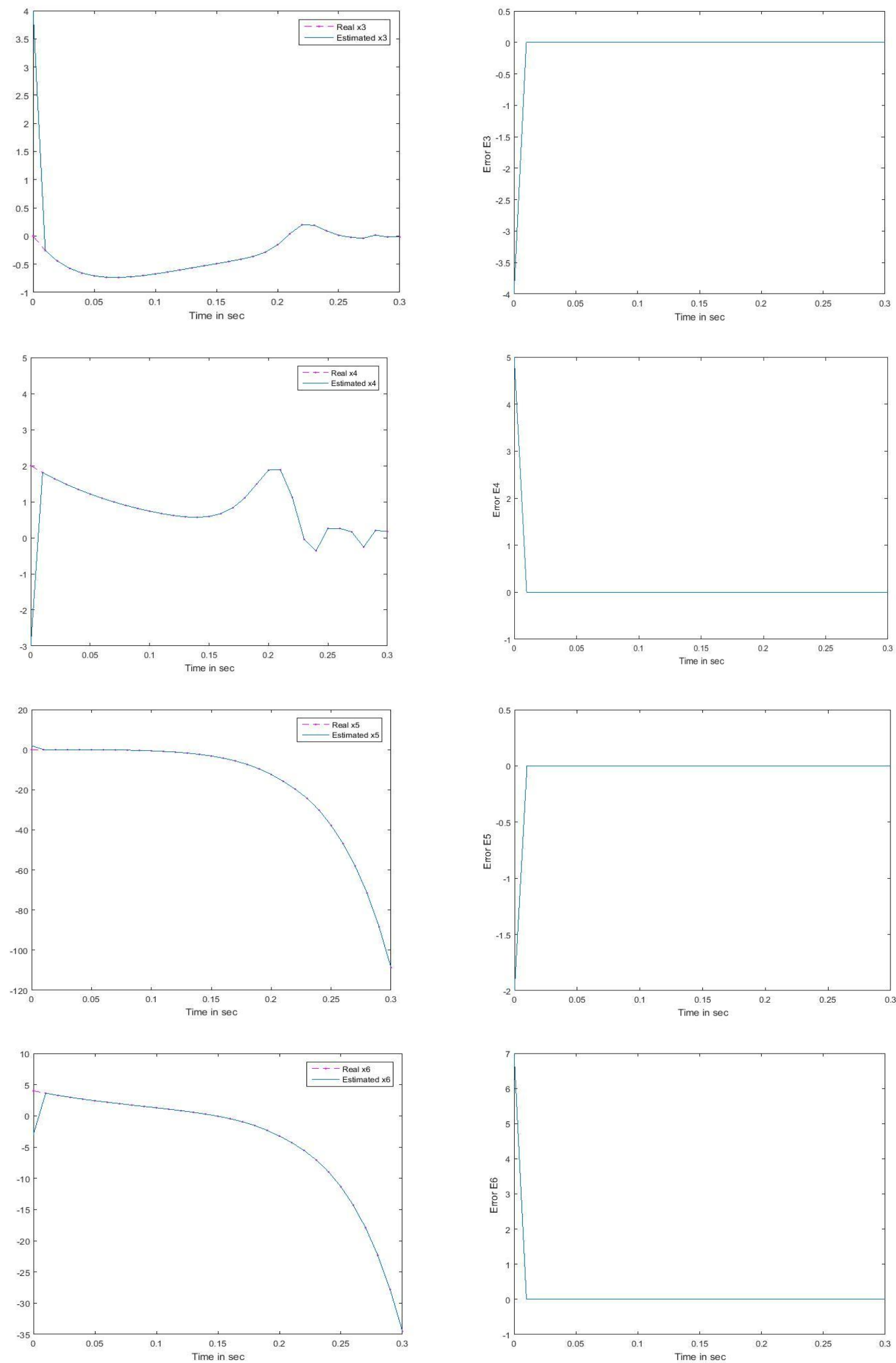

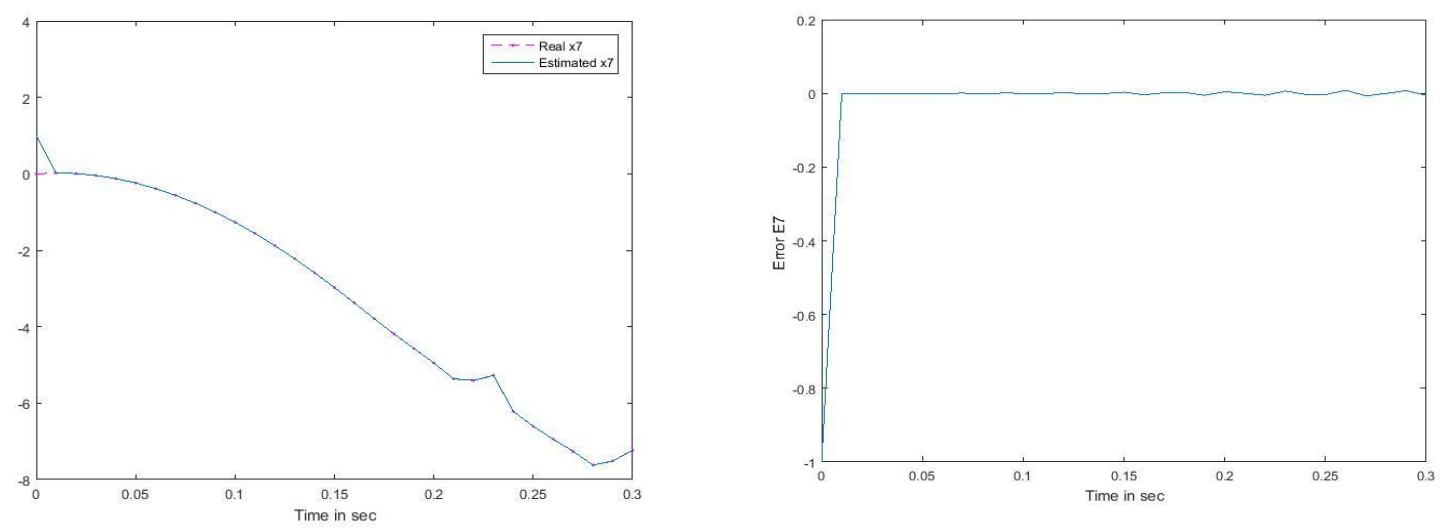

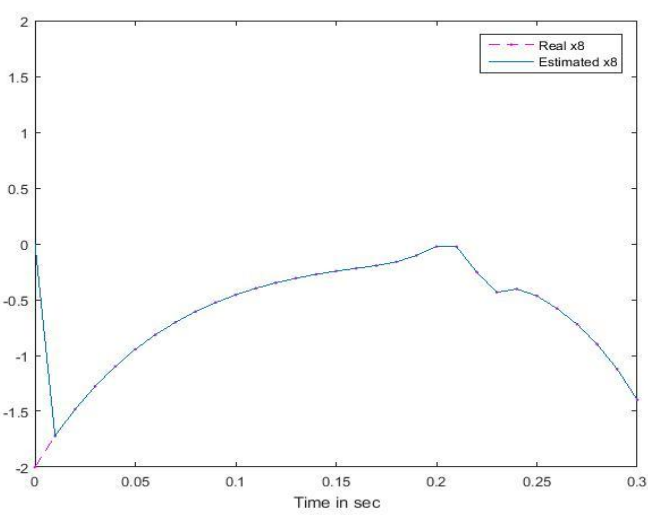

(a)

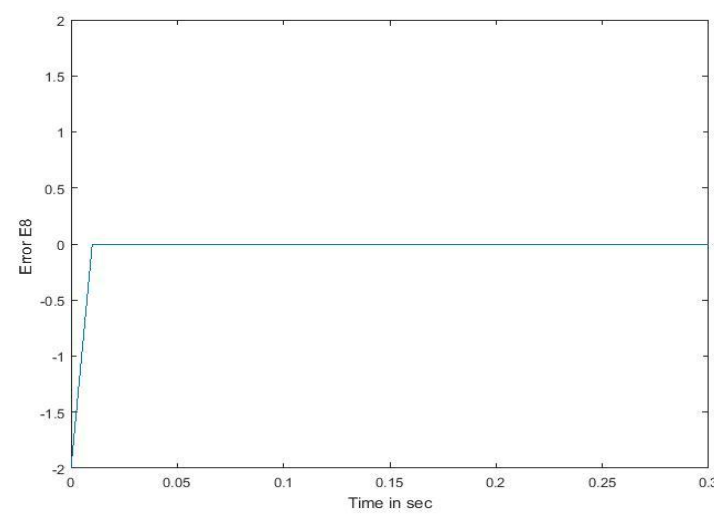

(b)

Fig 4:(a) Response Drive Synchronization Error of the 8D Hyperchaotic System $(\theta=5)$

(b) Synchronization Error $(\theta=5)$

\section{CHAOTIC MASKING}

This technique is considered the first proposal to use chaos to secure communication. It is presented in the references [23]. The principle is to scramble the message signal $m(t)$ in a chaotic signal $c(t)$ by a direct addition operation before transmitting it, to obtain an encrypted signal $s(t)$.

In order to recover the message signal at the authorized receiver, the same system generating the chaos is used both for transmission and reception, with the difference that in the receiver, this system is controlled by the received signal $r(t)$ (Equal to the signal $s(t)$ affected by the disturbances in the channel) to obtain the synchronization.

The order of magnitude of the message signal must be very low compared to that of the chaotic signal $c(t)$ as shown in fig. 5 to give no hope of retrieving it by the intruders without knowing the signal $c(t)$ and to have a good synchronization at the authorized receiver. 


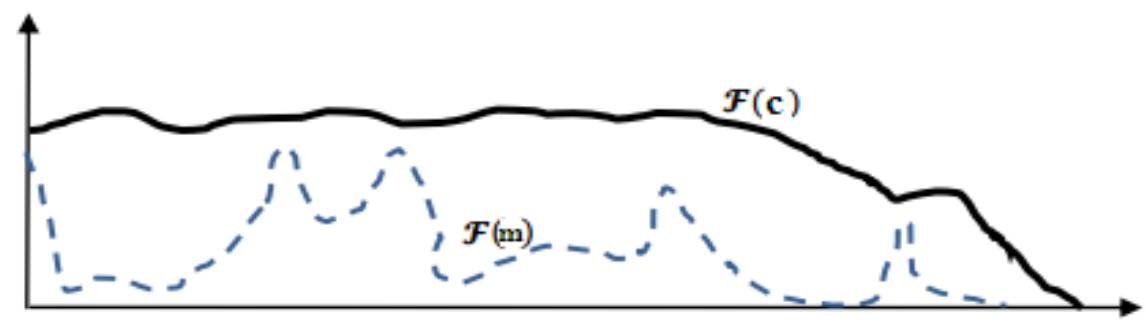

Fig 5: Spectrum of an information signal and chaotic signal

The encryption and decryption key is equal to the values of the coupling parameters between the transmitter and the receiver and the parameters characterizing the chaotic systems used.

Then the message signal is reconstituted by the difference between the received signal $r(t)$ and the signal $c^{\prime}(t)$ close to $c(t)$ result of the synchronization, see fig. 6 which illustrates this principle.

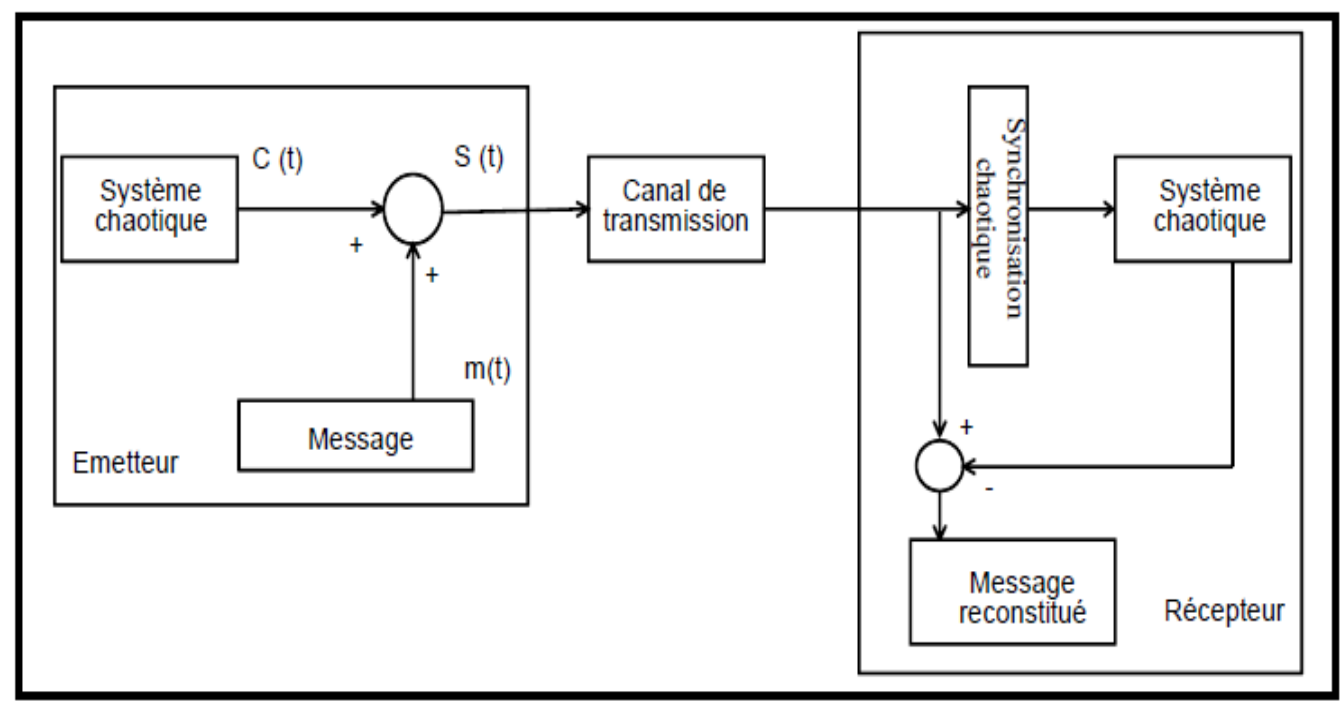

Fig 6: Schema of chaotic masking by addition

\subsection{Mounting of masking and synchronization}

In 1993, Cuomo proposed a much more interesting montage for this type of communication. Instead of transmitting two signals, it is possible to carry out an assembly with only the chaotic signal which will control the subsystem stable to the receiver. The signal will be masked by it with a small amplitude [24].

The subsystem was stable to the disturbances that would come from the information signal, it could be synchronized correctly. This assembly is illustrated in fig. 7 where the stable subsystem is $(y, z)$ and $x$ is the chaotic control signal. On the other hand, this technique requires the subsystem $x$ to be stable by being controlled by $(y, z)$. 


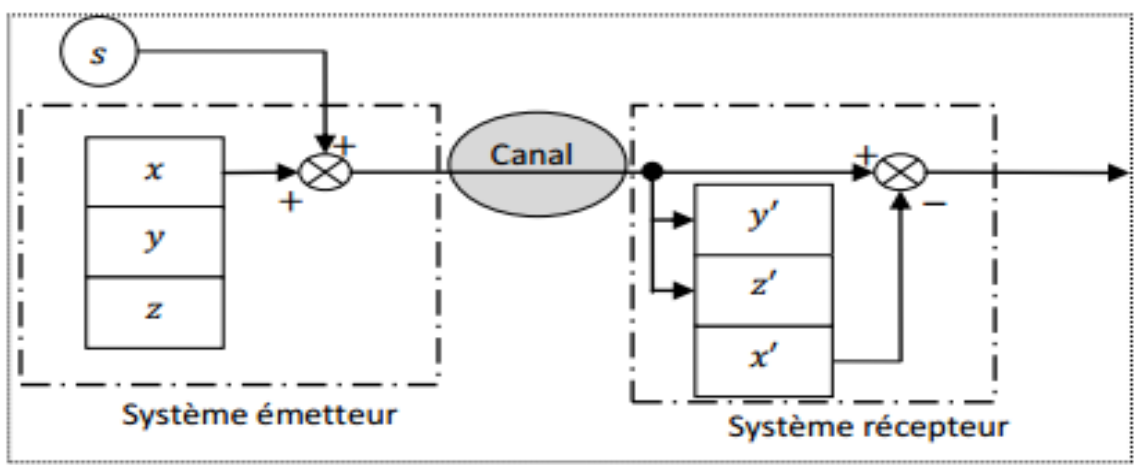

Fig 7: Mounting of Cuomo

This arrangement has the advantage of revealing less information about the nature of the system $(x, y, z)$ but the synchronization will obviously be less well so the recovered signal may be noisy. By choosing a sufficiently low amplitude ratio $m / x$ and having an $\mathrm{x}$ which has a fairly large and regular spectrum, this system can be quite effective for securing information. Thus, the receiver system in our case is the high gain observer.

\subsection{Simulation of hyperchaotic systems for additive masking}

Thus, in this simulation, we use the synchronization between two identical systems that we verified in section 2 . In this way the first master system is taken as the generator of a chaotic signal $\left(c(t)=x_{1}(t)\right)$ is a system state variable in the transmitter). At the receiver, the synchronization is done using the slave system (high gain observer) with the same initial conditions as those given in the transmitter, but controlled by the received signal $r(t)$.

Assuming that the perturbations due to the channel are negligible and that the transmission does not require modulation, we will then have:

$$
r(t)=s(t)=c(t)+m(t)
$$

fromwhere:

$$
s(t)=x_{1}(t)+m(t)
$$

With $m(t)$ the message signal presented in figure 8 .

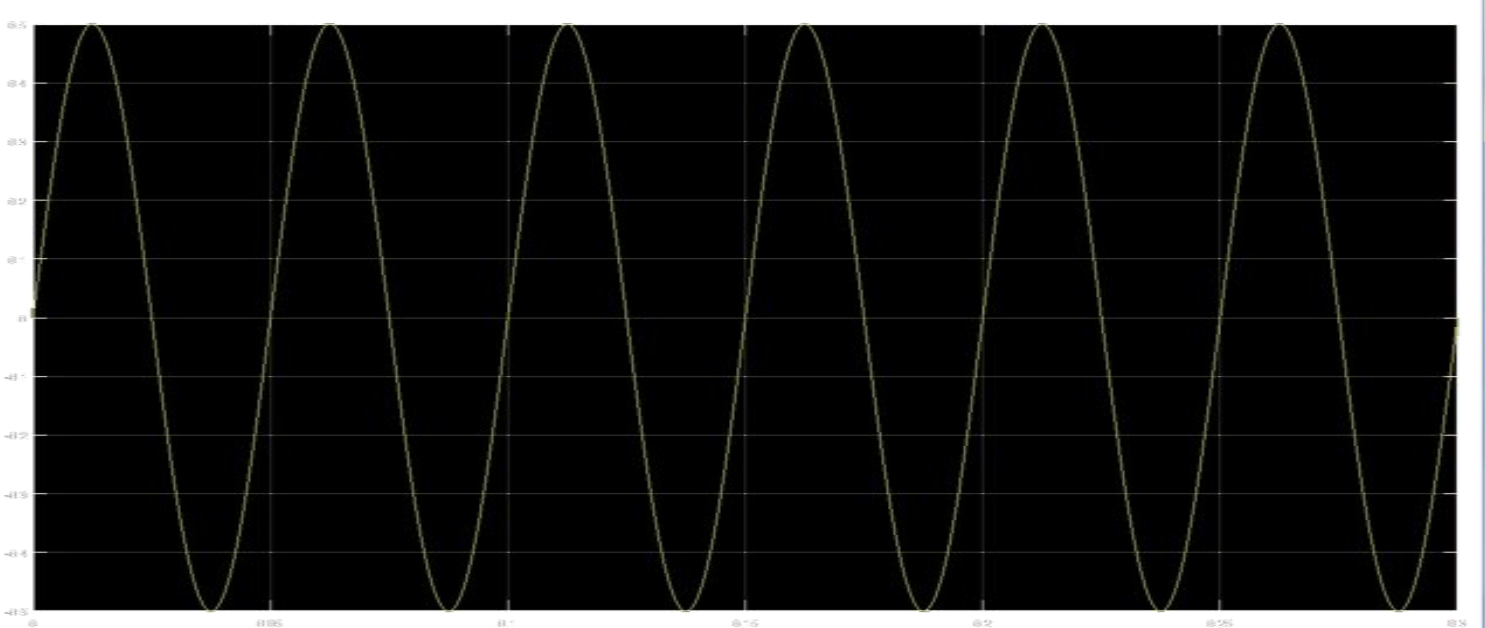

Fig 8 : Original message $m(t)$ 
The encryption key (or decryption key) is the parameters of the sending system. A sinusoidal signal with an amplitude equal to à 0.5 and a frequency equal to $10 \mathrm{~Hz}$ is chosen for $m(t)$. At the transmitter, the encrypted message will then be received and at the output of the receiver, the reconstituted message will then be received.

$$
\left\{\begin{array}{l}
\dot{\hat{x}}_{1}=-10 \hat{\boldsymbol{x}}_{1}+15 \hat{x}_{5}-\hat{x}_{3} \hat{x}_{5} \hat{x}_{6} \hat{x}_{8}-\theta * d_{\theta}^{-1} * S_{1}^{-1} * C^{\prime} C\left(\hat{x}_{1}-x_{1}\right) \\
\dot{\hat{x}}_{2}=0.5 \hat{x}_{2}-25 \hat{x}_{6}+\boldsymbol{S} \hat{x}_{4} \hat{x}_{5} \hat{x}_{8}-\theta * d_{\theta}^{-1} * S_{1}^{-1} * C^{\prime} C\left(\hat{x}_{2}-x_{2}\right) \\
\dot{\hat{x}}_{3}=-15 \hat{x}_{3}+15 \hat{x}_{8}-0.1 S \hat{x}_{2} \hat{x}_{4} \hat{x}_{6}-\theta * d_{\theta}^{-1} * S_{1}^{-1} * C^{\prime} C\left(\hat{x}_{3}-x_{3}\right) \\
\dot{\hat{x}}_{4}=-10 \hat{x}_{4}+\boldsymbol{S} \hat{x}_{2} \hat{x}_{3} \hat{x}_{5}-\theta * d_{\theta}^{-1} * S_{1}^{-1} * C^{\prime} C\left(\hat{x}_{4}-x_{4}\right) \\
\dot{\hat{x}}_{5}=20 \hat{x}_{5}+10 \hat{x}_{7}-S \hat{x}_{2} \hat{x}_{3} \hat{x}_{6}-\theta * d_{\theta}^{-1} * S_{1}^{-1} * C^{\prime} C\left(\hat{x}_{5}-x_{5}\right) \\
\dot{\hat{x}}_{6}=-10 \hat{x}_{6}+10 \hat{x}_{5}+\hat{x}_{2} \hat{x}_{3} \hat{x}_{4} \hat{x}_{7}-\theta * d_{\theta}^{-1} * S_{1}^{-1} * C^{\prime} C\left(\hat{x}_{6}-x_{6}\right) \\
\dot{\hat{x}}_{7}=5 \hat{x}_{7}+4 \hat{x}_{2}-1.5 S \hat{x}_{4} \hat{x}_{5} \hat{x}_{8}-\theta * d_{\theta}^{-1} * S_{1}^{-1} * C^{\prime} C\left(\hat{x}_{7}-x_{\grave{e}}\right) \\
\dot{\hat{x}}_{8}=-15 \hat{x}_{8}+\boldsymbol{S}-\hat{x}_{3} \hat{x}_{4} \hat{x}_{5} \hat{x}_{6}-\theta * d_{\theta}^{-1} * S_{1}^{-1} * C^{\prime} C\left(\hat{x}_{8}-x_{8}\right)
\end{array}\right.
$$

\subsection{Results and analysis}

The initial conditions of the 8D hyperchaotic system (9) are chosen as:

$$
\begin{array}{ccccc}
x_{1}=\hat{x}_{1}=0 & x_{2}=\hat{x}_{2}=1 & x_{3}=\hat{x}_{3}=0 & x_{4}=\hat{x}_{4}=2 \quad x_{5}=\hat{x}_{5}=0 \\
x_{6}=\hat{x}_{6}=4 & x_{7}=\hat{x}_{7}=0 & x_{8}=\hat{x}_{8}=-2
\end{array}
$$

The simulation results for an observer gain $\theta=5$ are as follows.

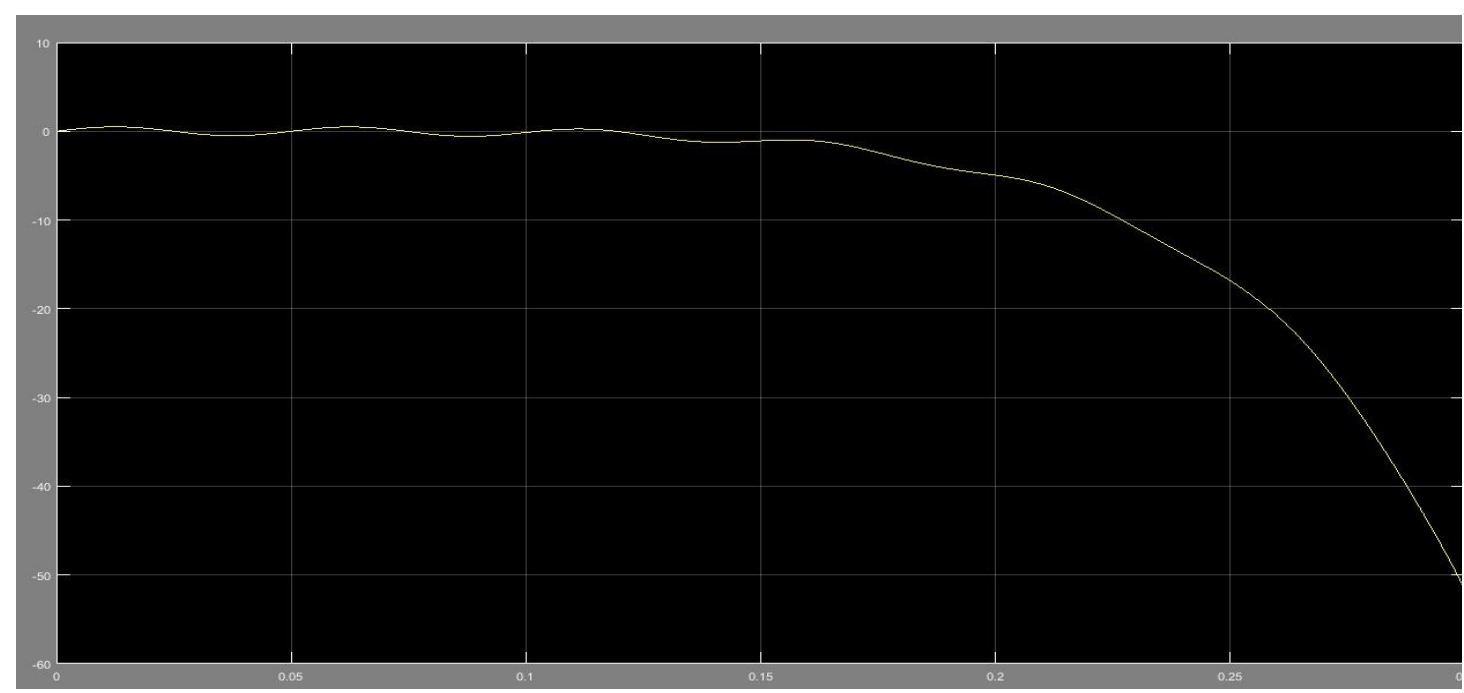

Fig 9: Encrypted message $s(t)$ of the new 8D hyperchaotic system 
DOI: https://dx.doi.org/10.26808/rs.ca.i7v5.03 International Journal of Computer Application (2250-1797)

Volume 7- No.5, September-October 2017

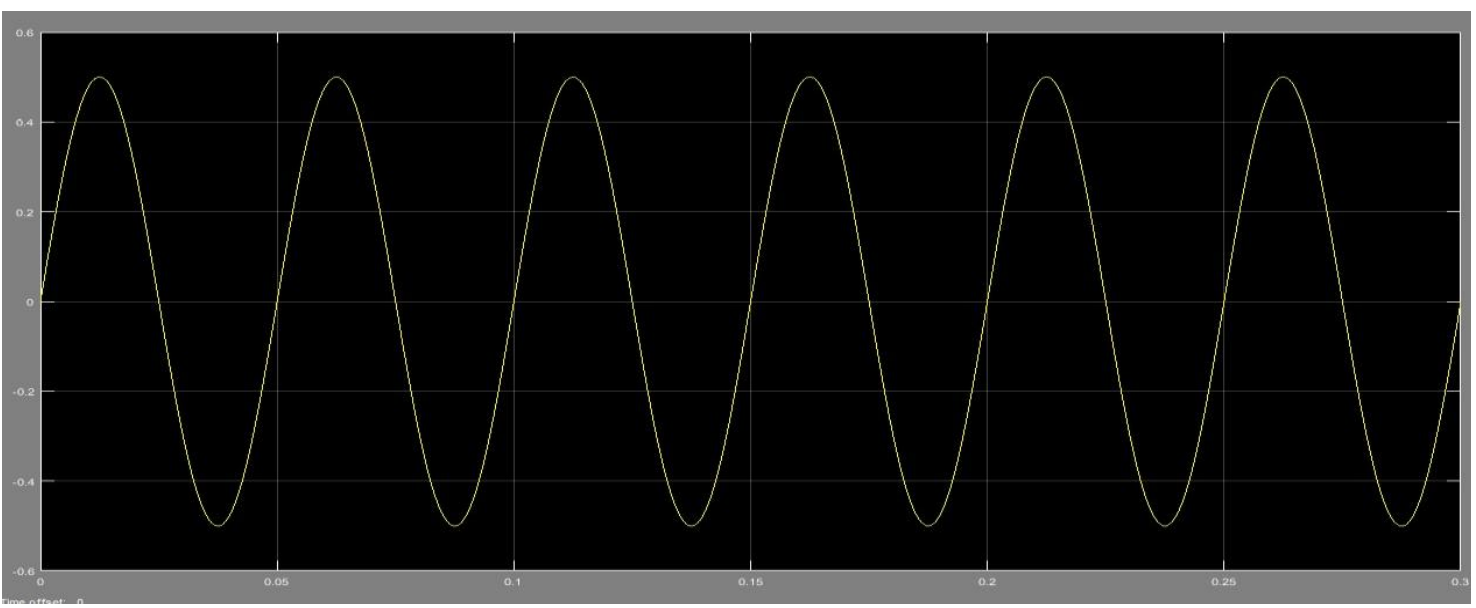

Fig 10: Restored message $m^{\prime}(t)$ by subtracting the state $x_{1}(t)$ from the observer of the $8 \mathrm{D}$ system

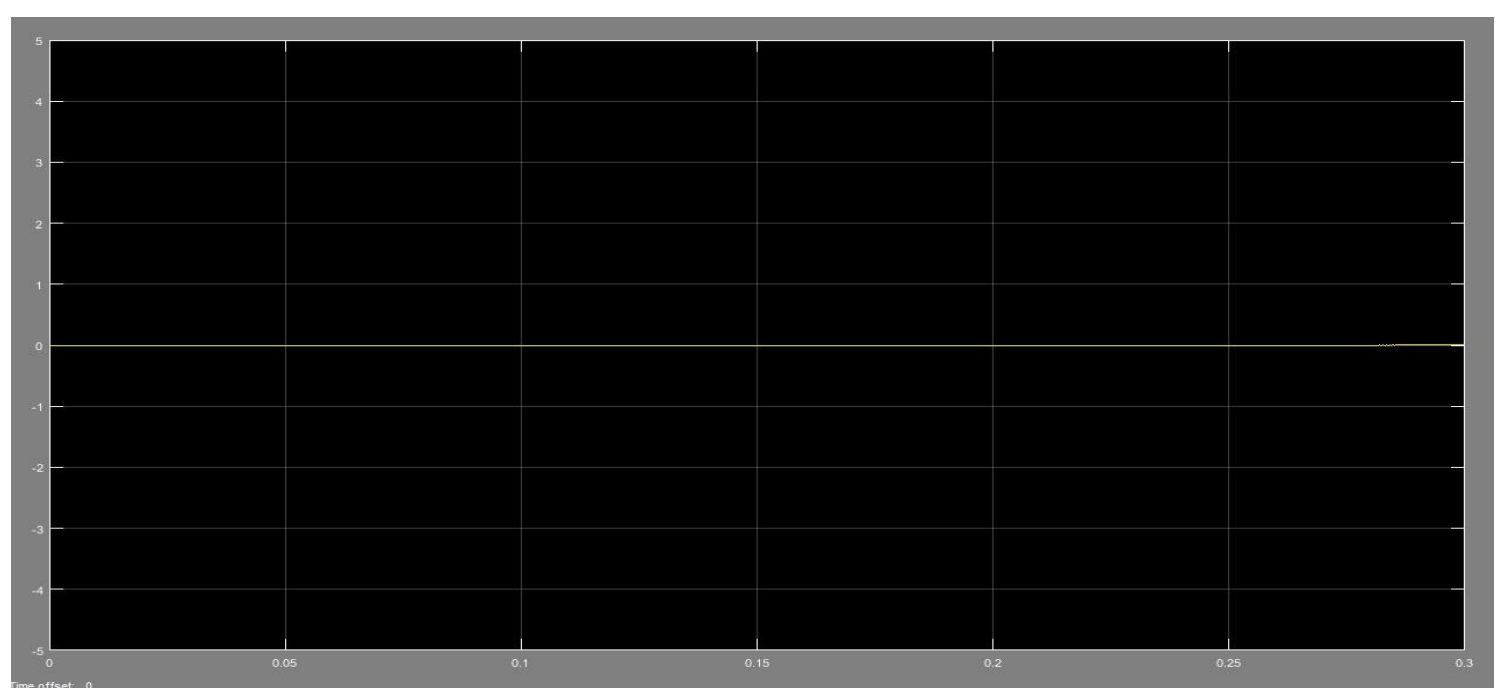

Fig 11: Synchronization error between $x_{1}(t)$ and $\hat{x}_{1}(t)$ of the $8 \mathrm{D}$ system

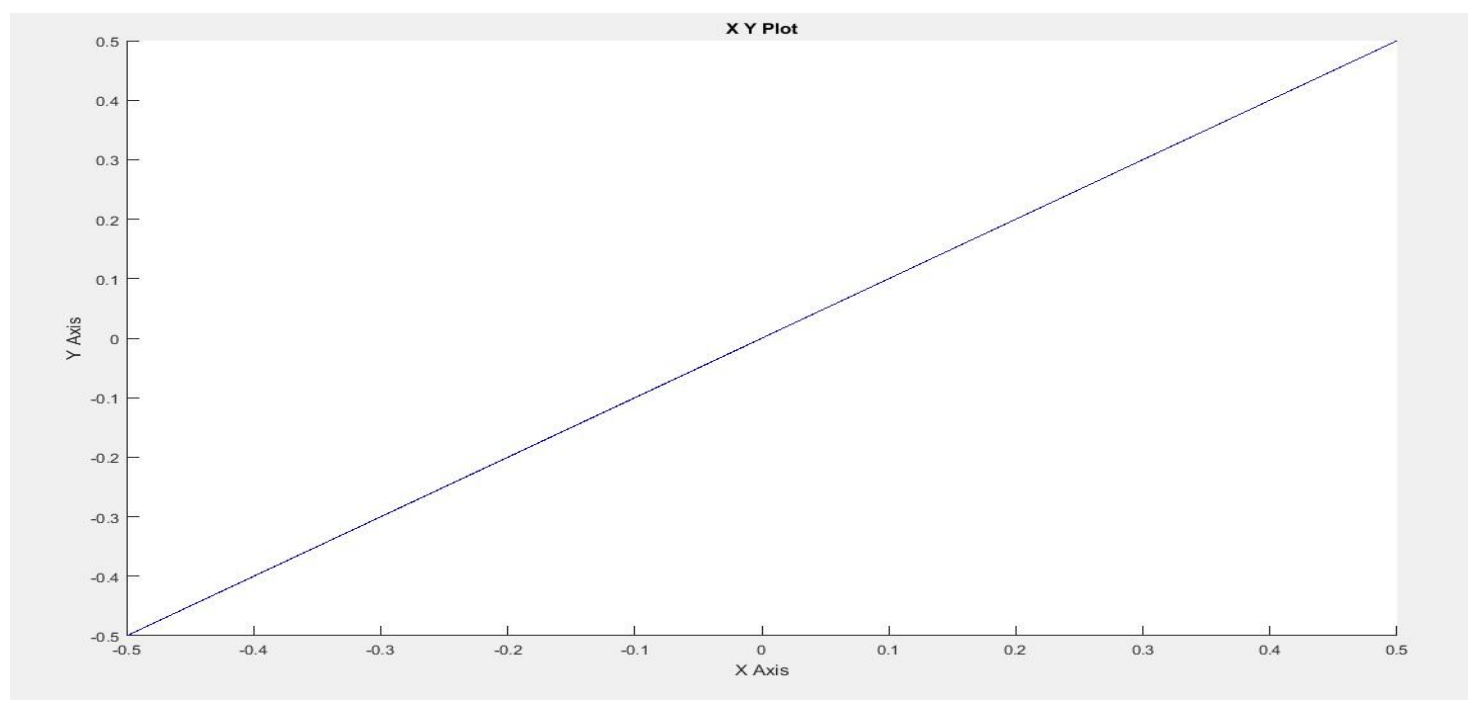

Fig 12: $m(t)$ as a function of $m^{\prime}(t)$ 
From the results shown in the figures above, for our studied hyperchaotic system, it can be seen that with the high-gain observer synchronization, it is possible to reconstruct the information signal with a rather low synchronization error.

\section{CONCLUSION}

Since the mathematical model reflects the real dynamics of our new eight dimensionalhyperchaotic system, this research paper presented the simulation of this hyperchaotic system with a certain initial conditions and parameters. This model is simulated by using MATLAB and SIMULINK.

From the simulation resultsof the hyperchaotic system studied, it is noted that with the synchronization by a high gain observer, that tend to zero when the observer gain grows, it is possible to reconstruct the information signal with a fairly low synchronization error. We remark that the reconstruction of the message signal is more perfect for the high order hyperchaotic signal.

\section{REFERENCES}

[1] A. Ali-Pacha, N. Hadj-Said, A. M'Hamed, A. Belghoraf. "Lorenz's Attractor Applied to the Stream Cipher (Ali- Pacha Generator)", Chaos, Solitons\&Fractals , Volume 33/5, pp.1762-1766, 2007.

[2] S.N.Lagmiri, M.Amghar, N.Sbiti, "Seven Dimensional New Hyperchaotic Systems: Dynamics and Synchronization by a High Gain Observer Design", International Journal of Control and Automation Vol. 10, No. 1 (2017), pp.251-266, Volume 10, No. 1, January 2017.

[3] LO .1, Yu S M, Leung H, Chen G. "Experimental Verification of Multi-directional Multiscroll Chaotic Attractors", IEEE Trans. Circuits and Systems I,Volume 53/1, pp.149-165, 2006.

[4] Simin Yu, Jinhu Lu, Leung, H., Guanrong Chen. "Design and Implementation of n-scroll Chaotic Attractors from a General Jerk Circuit[J]". IEEE Trans. Circuits and Systems I, Volume52/7, pp.1459-1476, 2005.

[5] Murali, K., Lakshmanan, M.: "Secure communication using a compound signal from generalized chaotic systems". Phys. Lett. A 241(6), 303-310 (1998)

[6] Zaher, A.A., Abu-Rezq, A.: "On the design of chaos-based secure communication systems". Commun. Nonlinear Syst. Numer. Simul. 16(9), 3721-3727 (2011)

[7] NiuYujun, Wang Mingjun, Zhang Huaguang. "A New HyperChaotic System and Its Circuit Implementation", Elsevier,Volume 15/11, pp.3518-3524, 2010.

[8] Rhouma, R., Belghith, S.: "Cryptoanalysis of a chaos based cryptosystem on DSP". Commun. Nonlinear Sci. Numer. Simul. 16(2), 876-884 (2011) 
[9] Feki, M.: "An adaptive chaos synchronization scheme applied to secure communication". Chaos Solitons Fractals 18(1), 141-148 (2003)

[10] Shi, J., Zhao, F., Shen, X., Wang, X.: "Chaotic operation and chaos control of travelling wave ultrasonic motor". Ultrasonics 53(6), 1112-1123 (2013)

[11] Rasappan, S., Vaidyanathan, S.: "Global chaos synchronization of WINDMI and Coullet chaotic systems by backstepping control". Far East J. Math. Sci. 67(2), 265-287 (2012)

[12] Usama, M., Khan, M.K., Alghatbar, K., Lee, C.: "Chaos-based secure satellite imagery cryptosystem". Comput. Math Appl. 60(2), 326-337 (2010)

[13] S.N. Lagmiri, H.ElMazoudi, N. Elalami, "Synchronization of 4-d hyperchaotic qi system by high gain observer", International Conference on Structural Nonlinear Dynamics and Diagnosis (CSNDD'2014), May 19-21, 2014. Agadir, Morocco.

[14] S.N.Lagmiri, M.Amghar, N.Sbiti, "Seven Dimensional New Hyperchaotic Systems: Dynamics and Synchronization by a High Gain Observer Design", International Journal of Control and Automation Vol. 10, No. 1 (2017), pp.251-266, Volume 10, No. 1, January 2017.

[15] Vaidyanathan, S.: "Sliding mode control based global chaos control of Liu-Liu-Liu-Su chaotic system". Int. J. Control Theor. Appl. 5(1), 15-20 (2012)

[16] S. Vaidyanathan and A.T. Azar, "Analysis, Control and Synchronization of a Nine-Term 3-D Novel Chaotic System", Chaos Modeling and Control Systems Design. Studies in Computational Intelligence, vol 581. Springer, Cham. (2015)

[17] Sundarapandian, V.: "Adaptive control and synchronization of uncertain Liu-Chen-Liu system". Int. J. Comput. Inf. Syst. 3(2), 1-6 (2011)

[18] Kengne, J., Chedjou, J.C., Kenne, G., Kyamakya, K.: "Dynamical properties and chaos synchronization of improved Colpitts oscillators". Commun. Nonlinear Sci. Numer. Simul. 17 (7), 2914-2923 (2012)

[19] S.N.Lagmiri, M.Amghar, N.Sbiti, "Hyperchaos to secure communications according to synchronization by a high gain observer", International Journal of Scientific Engineering and Technology, Volume No. 6, Issue No. 8, PP : 313-317, 1 Aug. 2017

[20] Sharma, A., Patidar, V., Purohit, G., Sud, K.K.: "Effects on the bifurcation and chaos in forced Duffing oscillator due to nonlinear damping". Commun. Nonlinear Sci. Numer. Simul. 17(6), 2254-2269 (2012)

[21] Sundarapandian, V.: "Output regulation of the Tigan system". Int. J. Comput. Sci. Eng. 3(5), 2127-2135 (2011)

[22] S.N.Lagmiri, M.Amghar, N.Sbiti, "Synchronization between a new chaotic system and Rössler system by a high gain observer", IEEEXplore, December 2014.

[23] L. Kocarev, K. S. Halle, K. Eckert, and L. O. Chua, Int. J. Bifurcation Chaos 2, 709, 1992.

[24] L. M. Pecora and T. L. Carroll, Phys. Rev. A 44, 2374, 1991 